\title{
Comparison of methods for determination of glomerular filtration rate: low and high-dose Tc-99m-DTPA renography, predicted creatinine clearance method, and plasma sample method
}

\author{
Majid Assadi - Mohammad Eftekhari · Mehrdad Hozhabrosadati • \\ Mohsen Saghari - Abdolali Ebrahimi - Iraj Nabipour · Mohammad Zaki Abbasi • \\ Darab Moshtaghi $\cdot$ Moloud Abbaszadeh $\cdot$ Sakineh Assadi
}

Received: 10 January 2008/Accepted: 23 July 2008/Published online: 9 August 2008

(C) Springer Science+Business Media B.V. 2008

\begin{abstract}
The gamma camera uptake method with Tc-99m-DTPA (diethylenetriaminepentaacetic acid) is a simple method for determination of glomerular filtration rate (GFR), and is less time-consuming than other methods, but its diagnostic accuracy is debated. Gate's method (low-dose; LD), the high-dose method (HD), the predicted-clearance method, and the plasmaclearance method with Tc-99m-DTPA are compared in this study. We also performed GFR measurement and diuretic renography simultaneously. Tc-99m DTPA renography was performed in 36 patients aged 18-72 years with a wide range of renal function (serum creatinine $1.37 \pm 0.49 \mathrm{mg} / \mathrm{dl}$ ). GFR was determined by four methods: the gamma camera uptake method with low-dose Tc-99m DTPA (Gates, LD); the gamma camera uptake method with high-dose Tc-99m DTPA (HD); the predicted creatinine clearance method (Cockcroft-Gualt, CG); and the plasma sample clearance (PSC) method using a mono-exponential curve. The
\end{abstract}

M. Assadi $(\bowtie) \cdot$ A. Ebrahimi · I. Nabipour

M. Z. Abbasi - D. Moshtaghi · M. Abbaszadeh .

S. Assadi

Department of Oncology and Nuclear Medicine, The Persian Gulf Tropical Medicine Research Center, Bushehr

University of Medical Sciences, Boostan 19 Alley,

7514763448, imam Khomeini Street, Bushehr, Iran

e-mail: assadipoya@yahoo.com

M. Eftekhari · M. Hozhabrosadati · M. Saghari Research Institute for Nuclear Medicine, Shariati Hospital, Tehran University of Medical Sciences, Tehran, Iran
PSC method was chosen as reference. The regression equations for the CG, Gates (low-dose), and HD methods against the PSC method were $28.68+0.80 \mathrm{X}$ ( $r=0.72 ; P$ value $<0.0001, \mathrm{RMSE}=21.65 \mathrm{ml} / \mathrm{min} /$ $\left.1.73 \mathrm{~m}^{2}\right), 6.19+0.79 X(r=0.90 ; P$ value $<0.0001$, $\left.\mathrm{RMSE}=10.64 \mathrm{ml} / \mathrm{min} / 1.73 \mathrm{~m}^{2}\right)$, and $6.53+0.88 X$ $(r=0.93 ; P$ value $<0.0001, \mathrm{RMSE}=9.35 \mathrm{ml} / \mathrm{min} /$ $1.73 \mathrm{~m}^{2}$ ), respectively. In comparison with determination of GFR by the PSC method, the CG method tended to overestimate GFR while, perversely, the LD and HD methods tended to underestimate GFR. The three methods were in agreement with the PSC method but the high-dose GFR method resulted in less error in estimation of GFR. Furthermore, GFR measurement and diuretic renography could be performed at the same time when the high-dose method was used. Because of the low cost and negligible radiation burden, this method might be preferred for routine practice in nuclear medicine.

Keywords Diuretic renogram - GFR ·

Tc-99m DTPA · Gates · Cockcroft-Gualt

\section{Introduction}

Glomerular filtration rate is a commonly accepted standard measure of renal function. The ideal way of assessing renal function is to measure the clearance of a substance that is freely filtered by 
glomeruli and does not undergo reabsorption or secretion. The generally accepted gold-standard technique for GFR assessment is inulin infusion [1]. This technique is difficult and time-consuming to perform and is therefore regarded as inappropriate for routine clinical use. Determination of GFR as measured by creatinine has been a recognized means of assessing renal function for many decades [2]. Procedures using radiopharmaceuticals, especially Tc-99m DTPA (diethylenetriaminepentaacetic acid), have been proposed as rapid, less invasive, and reliable options for estimating GFR [3]. Accurate determination of the plasma concentration of the injected radiotracer requires analysis of Tc- $99 \mathrm{~m}$ DTPA levels in multiple blood samples obtained over several hours and it is regarded as a reference method [4]. However, this procedure requires serial blood samples in order to perform the computation necessary to calculate GFR. Thus the time interval needed for acquiring the multiple blood samples, which may be up to $4 \mathrm{~h}$, combined with sample counting and subsequent back extrapolation of data to determine the radionuclide clearance rate, impose logistical constraints on a busy nuclear medicine department. Measuring clearance of Tc-99m DTPA using a single blood sample has led to less accurate results [5].

Camera-based methods for measuring renal uptake of Tc-99m DTPA without blood or urine sampling have been widely used [6]. The method introduced by Gates [7] has been the most common routine method $[8,9]$.

Although the diagnostic accuracy of these methods is debated, they are regarded as convenient and less invasive, and can also be used to evaluate the function of each kidney individually $[8,9]$. However, a simple and accurate determination of GFR is still a clinical challenge [10].

In addition, because the large dose of Tc- $99 \mathrm{~m}$ DTPA used in diuretic renography can induce significant camera dead-time errors in syringe counting, dosing for diuretic renography is not carried out simultaneously with GFR calculation and must be performed separately.

The aim of this study was to evaluate a camerabased method for estimation of GFR at the same time as obtaining a diuretic renogram, known as the high dose method (HD). Such a method would add convenience to evaluation of renal function in routine clinical practice. We also examined the relative accuracy of GFR determined by the Cockcroft-Gualt (CG), Gates, and HD methods and compared them with GFR obtained by using a sampling DTPA plasma-clearance method (as a reference).

\section{Materials and methods}

\section{Participants and study design}

Thirty-six patients who were referred to the department of nuclear medicine for evaluation of renal function were selected randomly for inclusion in this study. The patients were recruited from the urology and nephrology clinics at our hospital.

They were given a wide variety of clinical diagnoses including chronic renal failure in four patients, diabetic nephropathy in four, pyelonephritis in one, reduced renal function of unknown cause in four, pre-surgical renal function evaluation in 12, various others in the remaining 11 cases.

The GFR was measured in each patient by four separate methods. These methods were implemented to 36 subjects for whom renograms with split renal function accompanied by GFR were requested.

Determination of GFR by measurement of Tc-99m DTPA clearance in multiple blood samples was used as the reference method for evaluating renal function. The GFR results obtained with these methods were compared, and the correlation coefficient was determined.

This study was approved by the institutional ethics committee of Tehran University of Medical Science and all patients signed written informed consent.

\section{GFR methods}

Gates (low dose) method

Each patient was hydrated with $300 \mathrm{ml}$ water $20 \mathrm{~min}$ prior to the examination. The patient lay down on a bed in the supine position. All patients received 111 $\mathrm{MBq}$ Tc-99m DTPA as a rapid intravenous bolus over 5-10 s through a heparin well. Immediately after injection, the heparin well was flushed with $10 \mathrm{ml}$ sterile saline. A commercial Tc-99m-DTPA preparation (Atomic Energy Organization of Iran; AEOI, 
Tehran, Iran) was used. The labelling and qualitycontrol procedures of this preparation were performed according to the manufacturer's instructions. The activity of the syringe and injection catheter was measured before and after injection, and the postinjection value was subtracted from the pre-injection value to determine the net delivered activity. The activity was placed at a point $30 \mathrm{~cm}$ distant from detector on the imaging table and was counted for $1 \mathrm{~min}$. Immediately after injection of Tc-99m DTPA, posterior dynamic renal flow images (a 2-s frame for $60 \mathrm{~s}$ followed by a 30 -s frame for $5 \mathrm{~min}$ ) were obtained in a $128 \times 128$ matrix. Residual activity in the syringe was measured using a dose calibrator. The GFR was calculated using the activity in the region of the kidneys detected by the posterior detector between 2 and $3 \mathrm{~min}$ after injection of Tc-99m DTPA. Region of interest (ROI) over each kidney was assigned manually on the frame added after injection by selecting a crescent-shaped background ROI in the inferior regions of the kidneys (Fig. 1).
Renal depth was estimated using the Tønnesen equation at the level of the kidneys [7].

Renography, including high-dose method

The patient was hydrated with $300 \mathrm{ml}$ water $20 \mathrm{~min}$ prior to the examination and lay down on a bed in the supine position. Tc-99m-DTPA was given through an indwelling butterfly needle in an antecubital vein and was followed by infusion of $20 \mathrm{ml}$ normal saline. After counting 18.5 MBq on a dual-headed gamma camera (ADAC Genesys Malpitas, CA, USA) equipped with a pair of low-energy collimators, $5.18 \mathrm{MBq} / \mathrm{kg}$ Tc-99m-DTPA administered to each case. After that the ratio of injected dose (renogram) to $18.5 \mathrm{MBq}$ of Tc-99m-DTPA was calculated. The ratio of multiplied to acquired counts with $18.5 \mathrm{MBq}$ Tc-99m-DTPA was inserted in the Gate formula as pre-syringe count.

Frames of $128 \times 128$ matrix were recorded with an online-computer, initially at one-second intervals

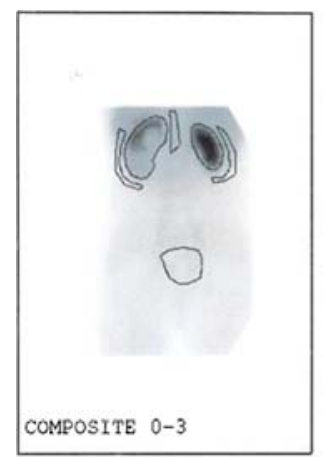

FLOW IMAGES TIMING:
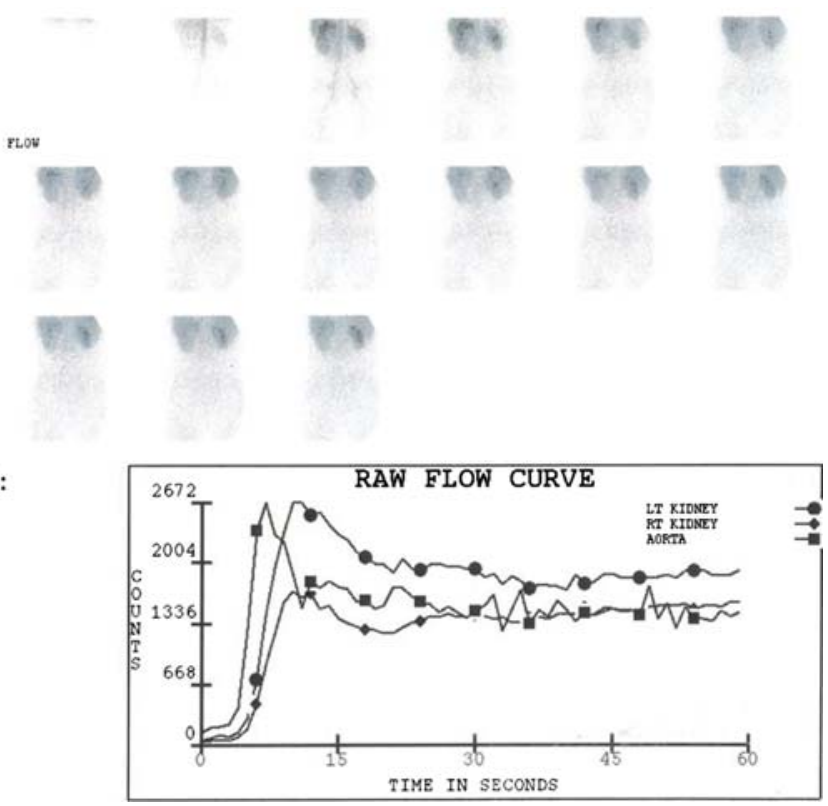

Fig. 1 Dynamic phase of Tc-99m DTPA renogram shows prompt perfusion to the right kidney and delayed perfusion to the left. On sequential static images, there is prompt accumulation of tracer in the right intrarenal collection system with subsequent excretion into ureter and bladder. Early images show central photopenic region in the left kidney which gradually fills with urinary tracer, but dose not empty. Time-activity curves demonstrate normal time-to-peak on the right and prompt washout of urinary tracer in response to lasix.
There is delayed time-to-peak on the left with no significant urinary tracer washout following lasix. In addition, the camera based GFR methods was quanitified using a dual-detector gamma camera and activity between 2 and $3 \mathrm{~min}$ after injection. A semilunar background is drawn in the inferior of each kidney. Individual GFR $(\mathrm{ml} / \mathrm{min})$ and the percentage of differential function then can be calculated. Furthermore, two other methods (CG and PSC) were also carried out 
for $1 \mathrm{~min}$ and then at ten-second intervals for $29 \mathrm{~min}$. The post-injection syringe with a straight needle which was detached before the injection was again counted in the same way as for pre-injection.

The region of interest (ROI) over each kidney was assigned manually on the frame added from 2 to 3 min after injection. The GFR (GFRHD) was automatically estimated by use of a commercially available computer (ADAC Genesys Malpitas, CA, USA) according to the Gates' algorithm. Two nuclear medicine specialists and three nuclear medicine technicians were involved in the data analysis.

Venous blood samples were obtained from the arm contralateral to the injection site $60,120,180,240$, and $300 \mathrm{~min}$ after injection of Tc-DTPA. The samples were centrifuged, and plasma activity was measured in a well counter (DCM-200, Aloka, Tokyo, Japan).

\section{Plasma-clearance method}

Radioactivity in the plasma (ml) was calculated directly, without a dilution procedure of a standard injected solution, by the well counter. The GFR (GFRpsc) was determined $60,120,180,240$, and 300 min postinjection. The GFR was estimated from a monoexponential model fitted to multiple venous blood samples, from a single exponential curve fitted through points corresponding to multiple plasma samples [11-13].

Predicted creatinine clearance (Cockcroft-Gault's method)

The GFR (GFRcg) was also predicted from the serum creatinine (SCr) level at renography using the Cockcroft-Gault's equation [14]:

For men GFR $(\mathrm{ml} / \mathrm{min})=[(140-$ age $) \times$ weight $] /$ $(\mathrm{SCr} \times 72)$. For women: $\mathrm{GFR}(\mathrm{ml} / \mathrm{min})=0.85 \times[(140-$ age) $\times$ weight $) /(\mathrm{SCr} \times 72)$, where weight is body weight $(\mathrm{kg})$ and $\mathrm{SCr}$ is serum creatinine level $(\mathrm{mg} / \mathrm{dl})$

The serum creatinine was measured by use of an autoanalyser (Olympus AU-602, Tokyo, Japan) with an enzyme method.

\section{Normalization of GFR}

The GFR ( $\mathrm{ml} / \mathrm{min}$ ) obtained by the four methods was normalized for a body surface area of $1.73 \mathrm{~m}^{2}$ according to Haycock's equation [15].

\section{Statistical analysis}

For method comparison, standard linear least-squares regression analysis and correlation test were used. Data are presented as the mean \pm one standard deviation. For these analyses, commercially available statistical product and service solutions (SPSS ver.13; SPSS, Tokyo, Japan) and Microsoft Office Excel 2003 with added Analyse-it software were used.

\section{Results}

The study included 21 males and 15 females with mean age $45.42 \pm 17.18$ and $47.13 \pm 14.57$ years, respectively. There was no significant difference in age of the two genders $(P$ value $=0.75)$. Measured $\mathrm{SCr}$ (normal $\leq 1.0 \mathrm{mg} / \mathrm{dl}$ ) for the subjects ranged from $0.50 \mathrm{mg} / \mathrm{dl}$ to $2.24 \mathrm{mg} / \mathrm{dl}$ with mean $1.37 \mathrm{mg} / \mathrm{dl}$ $(\mathrm{SD}=0.49 \mathrm{mg} / \mathrm{dl})$.

The values of GFR with the PSC method were $64.89 \pm 30.91$ and $53.63 \pm 21.36 \mathrm{ml} / \mathrm{min} / 1.73 \mathrm{~m}^{2}$ for males and females, respectively, which was not statistically significantly different $(P$ value $=0.23$ ).

The regression equations of the CG, Gates (low dose), and the HD methods against the PSC method were $28.68+0.80 X \quad(r=0.72 ; P$ value $<0.0001$, RMSE $\left.=21.65 \mathrm{ml} / \mathrm{min} / 1.73 \quad \mathrm{~m}^{2}\right), \quad 6.19+0.79 X$ $(r=0.90 ; P$ value $<0.0001$, RMSE $=10.64 \mathrm{ml} / \mathrm{min} /$ $\left.1.73 \mathrm{~m}^{2}\right)$, and $6.53+0.88 X(r=0.93 ; P$ value $<$ $0.0001, \quad$ RMSE $=9.35 \mathrm{ml} / \mathrm{min} / 1.73 \mathrm{~m}^{2}$ ) respectively (Figs. 2-4).

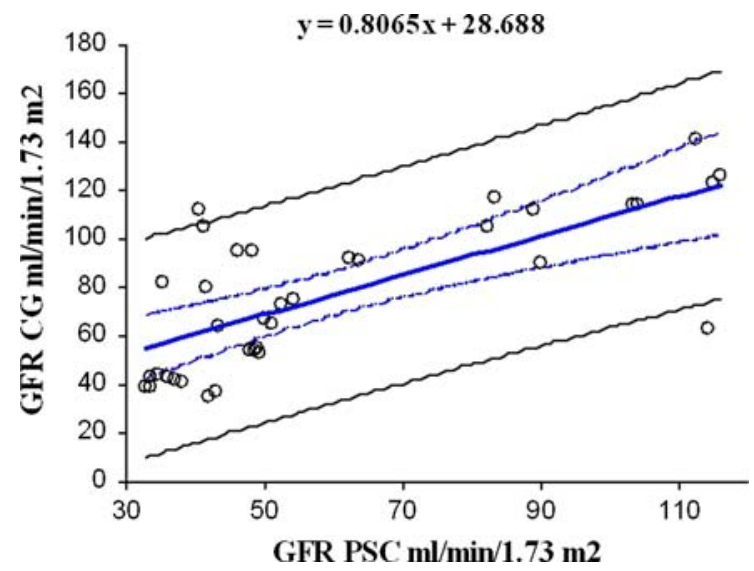

Fig. 2 Linear regression of the reference glomerular filtration rate (GFRpsc) versus the CG glomerular filtration rate (GFRCG) in 36 subjects. The dotted curves represent the 95\% tolerance limits of GFRpsc for a given GFRCG 


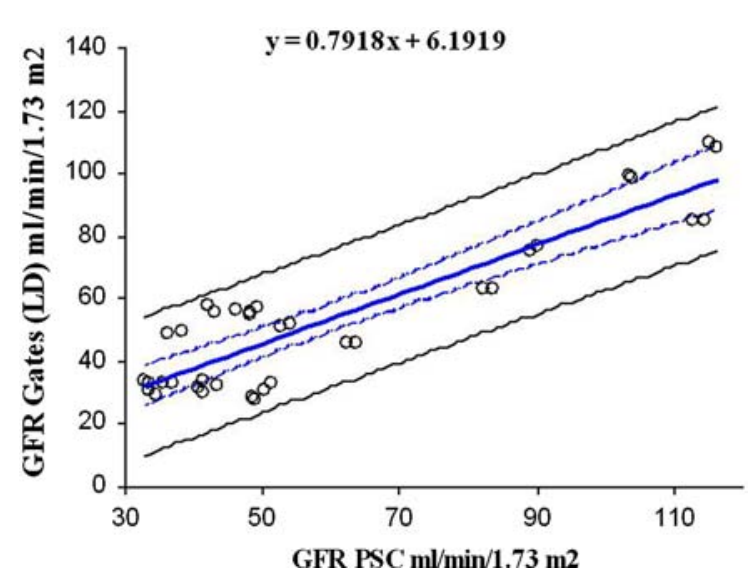

Fig. 3 Linear regression of the reference glomerular filtration rate (GFRpsc) versus the low dose glomerular filtration rate (GFRLD) in 36 subjects. The dotted curves represent the $95 \%$ tolerance limits of GFRpsc for a given GFRLD

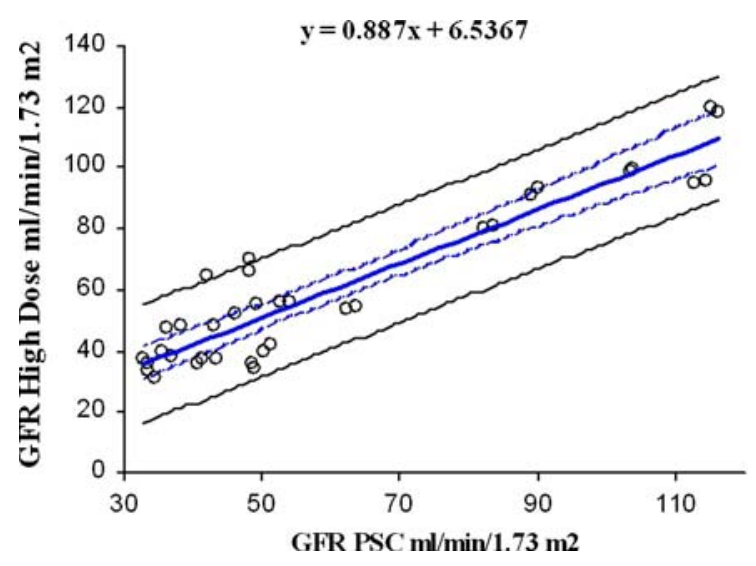

Fig. 4 Linear regression of the reference glomerular filtration rate (GFRpsc) versus the $\mathrm{HD}$ glomerular filtration rate (GFRHD) in 36 subjects. The dotted curves represent the 95\% tolerance limits of GFRpsc for a given GFRHD

Table1 Differences between GFR measured by the CG, lowdose, and high-dose methods against the plasma sample method

\begin{tabular}{lrrrr}
\hline Method & \multicolumn{1}{l}{ Mean } & \multicolumn{1}{l}{ SD } & Max & \multicolumn{1}{l}{ Min } \\
\hline CG & -17.03 & 22.00 & 51.40 & -71.70 \\
Low-dose & 6.33 & 11.96 & 29.20 & 15.70 \\
High-dose & 0.26 & 9.73 & 19.20 & -22.40 \\
\hline
\end{tabular}

The Gates (low-dose) method tended to underestimate GFR while the CG method had tendency to overestimate GFR (Table 1, Fig.5). Differences between the GFR (GFRpsc - GFRcg), (GFRpsc GFRgates), and (GFRpsc - GFRHD) were $-17.30 \pm$

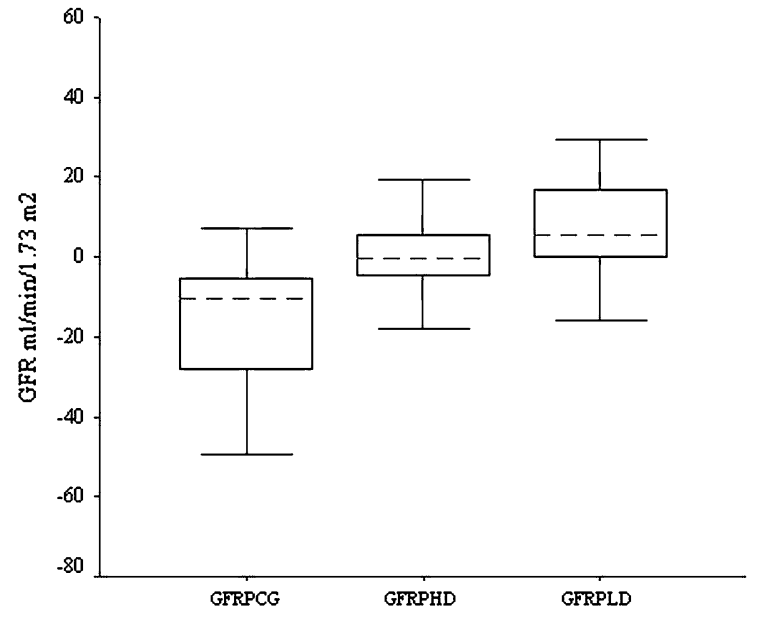

Fig. 5 Boxplots of difference in GFRs by the CockcroftGault's, High dose and Gates' method (Low Dose) against the PSC GFR method. The dotted lines indicate the median and the continuous line quartiles values. Difference in the GFRpscGFRCG \# GFR CG; Difference in the GFRpsc-GFRHD \# GFR HD; Difference in the GFRpsc-GFRLD \# GFR LD

$22.00 \mathrm{ml} / \mathrm{min} / 1.73 \mathrm{~m}^{2}, 6.33 \pm 11.96 \mathrm{ml} / \mathrm{min} / 1.73 \mathrm{~m}^{2}$, and $\quad 0.26 \pm 9.73 \mathrm{ml} / \mathrm{min} / 1.73 \mathrm{~m}^{2}$, respectively (Table 1, Fig. 4).

\section{Discussion}

Accurate measurement of total or differential renal function plays an important role in the clinical management of various renal diseases. The inulin clearance method, which is known as the gold standard, requires constant infusion of inulin and accurately timed collection of blood and urine samples [1].

In clinical practice, plasma creatinine is measured as an estimate of the GFR, on the assumption that creatinine is completely filtered across the glomerulus and that creatinine production and excretion is constant. The plasma creatinine concentration is then inversely related to the GFR. In addition, creatinine production depends on muscle mass and is age and sex-related. To apply the $\mathrm{C} \& \mathrm{G}$ formula, plasma creatinine must be in the steady state [14]. The formula is inaccurate in patients with liver disease, muscle wasting, oedema or extreme adiposity [14]. Furthermore, with creatinine, the ratio of glomerular filtration to tubular excretion varies unpredictably in pathologic states [16]. Moreover, creatinine clearance 
determines the total glomerular filtration rate (GFR) and not individual renal function.

In our study, although the CG method was correlated with the PSC method $(r=0.72)$ it was less precise than the Gates method for predicting the GFR. In addition, the CG method tended to overestimate GFR which is explained by the above reasons and has been shown in prior studies [17].

The Gates (LD) method correlated well with the plasma sample method $(r=0.91)$ and was more accurate than the CG method. In addition, the Gates (LD) method underestimated GFR values. These results were consistent with previous results $[18,19]$. Galli et al. demonstrated that the Gates' method (LD) underestimated GFR at all levels between 25 and $150 \mathrm{ml} / \mathrm{min}$ in 40 adult patients in comparison with Sapirstein's formula and Russell's two-sample method using ${ }^{51} \mathrm{Cr}$-EDTA [20]. In another study, by Wang et al., comparison of the Gates (LD) and 24-h $\mathrm{CCr}$ methods for GFR determination showed a correlation coefficient $(r)$ of 0.89 [21]. Despite the relative accuracy of the Gates method, others have not reported GFR values with the same accuracy [4, 22].

Gates reported a good correlation between renal uptake of DTPA and renal function using height and weight to measure renal depth (Tønnesen formula) [7].

Gates' method (LD) estimates the clearance based on the ratio of the sum of the left and right renal count rates approximately 2 min post-injection to the estimated dose measured in kidney geometry in the posterior view. This ratio depends on several situations and on a number of measured and estimated conditions, for example net injected dose, system dead time, the quality of the injection bolus, estimated kidney depths based on patient height and weight (particularly critical in obese patients), gamma camera sensitivity, linear attenuation coefficient of the radioisotope in the body at the level of the kidneys, and the sizes and shapes of the manually or automatically created whole kidney and renal background regions of interest (ROIs) in the individual patient. We used routine quality-control testing of the DTPA after reconstitution and also corrected background activity by selecting a crescent-shaped background region of interest (ROI) in the inferior regions of kidneys to prevent errors in the estimation of GFR.

Our data showed that the high-dose method can be an acceptable method of determining GFR and obtaining a diuretic renogram simultaneously.
The high-dose method correlated better than the low-dose method with PSC GFR. This may be because of greater count loss in most gamma camerasbecause we counted $18 \mathrm{MBq}$ as a presyringe injection in the HD method compared with $111 \mathrm{MBq}$ in the LD method-and, second, because administration of about $370 \mathrm{MBq}$ in the $\mathrm{HD}$ method rather than 111 MBq in the LD method results in better contrast and, subsequently, better drawing of kidney and background ROIs resulting from augmented signal-tonoise ratio ( $S / N$ ratio). We also considered the abovementioned utilized points in the acquisition and processing stages in the HD procedure.

A study similar to ours that was performed with 370-550 MBq (10-15 mCi) of 99mTc-DTPA and a gamma camera on 104 patients to compare this method and blood sampling for GFR assays resulted in a regression equation $y=6.9+0.91 x(r=0.94)$, and it was concluded that this method would be suitable for inter-institutional comparison and for longitudinal patient studies [23]. Mulligan et al. compared results of GFR computed using the HD technique with a six-point dual-exponential plasma reference model. A large dose (740 MBq) of Tc-99mDTPA was injected. The technique for determination of injected counts was modified to use a dose calibrator to measure the activity being injected, which, in turn, was multiplied by the camera counting efficiency to determine equivalent "camera count". The overall correlation $(r)$ was 0.895 [24].

Therefore, in addition to Tc-99m-DTPA renography enabling precise measurement of global and individual renal function, it provides notable information such as quantitative individual renal function and pathophysiological changes of the kidney in renovascular hypertension, hydronephrosis, and renal transplant.

Furthermore, GFR measurement and diuretic renography could be performed at the same time when the high-dose method is used. Because of low cost and negligible radiation burden, this method might be preferred for routine practice in nuclear medicine.

Finally it should be emphasized that our study has some drawbacks. In our study we calculated GFR as measured by creatinine using Cockcroft-Gault's method in few cases with wide ranges of creatinine and without consideration of some exclusion criteria for using the creatinine clearance method. It is, therefore, necessary to perform larger, well-designed studies. 


\section{Conclusion}

The three methods were in agreement with the PSC method but the high-dose GFR method resulted in less error in estimation of GFR. Gates' method is more precise than Cockcroft-Gault's method.

Because of its rather low costs, easy performance, and high accuracy, the HD method can be used safely, which may allow it to be easily performed as an adjunct to Tc-99m-DTPA renography, thereby providing clinicians with simultaneous measurement of GFR and a Tc-99m-DTPA renogram.

Acknowledgements This study was supported by Tehran University of Medical Sciences. We are indebted to Dr Jan Mohammad Malek Zadeh for his fruitful and useful suggestion. The authors thank the technologists at our department for data acquisition and other technical support.

\section{References}

1. Delpassand ES, Homayoon K, Madden T et al (2000) Determination of glomerular filtration rate using a dualdetector gamma camera and the geometric mean of renal activity: correlation with the Tc-99m DTPA plasmaclearance method. Clin Nucl Med 25(4):258-262. doi: 10.1097/00003072-200004000-00004

2. Yasui T, Itoh Y, Kojima Y et al (2008) Impact of microwave tissue coagulation during laparoscopic partial nephrectomy on postoperative renal function. Int Urol Nephrol 40(2):277-282. doi:10.1007/s11255-007-9271-1

3. Stacy BD, Thorburn GD (1966) Chromium-51 ethylenediaminetetraacetate for estimation of glomerular filtration rate. Science 152(725):1076-1077. doi:10.1126/science.152. 3725.1076

4. Itoh K (2003) Comparison of methods for determination of glomerular filtration rate: Tc-99m-DTPA renography, predicted creatinine clearance method and plasma sample method. Ann Nucl Med 17(7):561-565

5. Blaufox MD, Aurell M, Bubeck B et al (1996) Report of the Radionuclides in Nephrourology Committee on renal clearance. J Nucl Med 37(11):1883-1890

6. Rehling M, Jensen JJ, Sherling B et al (1989) Evaluation of renal function and morphology in children by $99 \mathrm{~m}$ Tc-DTPA gamma camera renography. Acta Paediatr Scand 78(4):601-607. doi:10.1111/j.1651-2227.1989.tb17944.x

7. Gates GF (1983) Split renal function testing using Tc-99m DTPA A rapid technique for determining differential glomerular filtration. Clin Nucl Med 8(9):400-407. doi:10.1097/ 00003072-198309000-00003

8. Shore RM, Koff SA, Hayes JR et al (1984) Glomerular filtration rate in children: determination from the Tc- $99 \mathrm{~m}$ DTPA renogram. Radiology 151(3):627-633

9. De Santo NG, Anastasio P, Cirillo M et al (1999) Measurement of glomerular filtration rate by the
99mTc-DTPA renogram is less precise than measured and predicted creatinine clearance. Nephron 81(2):136-140. doi: $10.1159 / 000045268$

10. Swan SK (1997) The search continues-an ideal marker of GFR. Clin Chem 43(6 Pt 1):913-914

11. Hilson AJW, Mistry RD, Maisey MN (1976) Tc 99mDTPA for the measurement of glomerular filtration rate. $\mathrm{Br}$ J Radiol 49:794-796

12. Kempi V, Persson RBR (1974) Tc 99m-DTPA(Sn) dry-kit preparation. Quality control and clearance studies. Nucl Med (Stuttg) 13:389-399

13. Chantler C, Garnett ES, Parsons V et al (1969) Glomerular filtration rate measurement in man by the single injection methods using 51Cr-EDTA. Clin Sci 37(1):169-180

14. Wieczorowska-Tobis K, Niemir ZI, Guzik P et al (2006) Difference in estimated GFR with two different formulas in elderly individuals. Int Urol Nephrol 38(2):381-385. doi:10.1007/s11255-006-0065-7

15. Haycock GB, Schwartz GJ, Wisotsky DH (1978) Geometric method for measuring body surface area: a heightweight formula validated in infants, children and adults. J Pediatr 93(1):62-66. doi:10.1016/S0022-3476(78)80601-5

16. Hoek FJ, Kemperman FA, Krediet RT (2003) A comparison between cystatin $\mathrm{C}$, plasma creatinine and the Cockcroft and Gault formula for the estimation of glomerular filtration rate. Nephrol Dial Transplant 18(10):2024-2031. doi:10.1093/ ndt/gfg349

17. Jones J, Burnett PC (1974) Creatinine metabolism in humans with decreased renal function. Clin Chem 20(9):1204-1212

18. Durand E, Prigent A, Gaillard J (1997) Comparison between 9 methods for estimation of glomerular filtration rate (GFR) with simultaneous injection of 51Cr-EDTA and 99mTc-DTPA. In: Taylor A Jr, Nally J, Thomsen H (eds) Radionuclides in nephrourology. Society of Nuclear Medicine, Reston, Virginia

19. Tønnesen KH, Munch O, Hald T et al (1974) Influence in the radiorenogram of variation in skin to kidney distance and the clinical importance hereof. In: Zum Winkel K, Blaufox MD, Funck-Brentano JL (eds) Radionuclides in nephrology. George Thieme, Berlin

20. Galli G, Rufini V, Vellante C et al (1997) Estimation of glomerular filtration rate with 99Tc(m)-DTPA: a comparative assessment of simplified methods. Nucl Med Commun 18(7):634-641. doi:10.1097/00006231-19970 7000-00007

21. Wang JY, Lu YS, Wang SJ et al (1995) Comparison and correlation of measurements of glomerular filtration rates by Tc-99m DTPA and 24-hour creatinine clearance. Zhonghua Yi Xue Za Zhi (Taipei) 55(6):432-437

22. Bratt CG, Larsson I, White T (1981) Scintillation camera renography with Tc 99m-DTPA and 131I-Hippuran. Scand J Clin Lab Invest 41(2):189-197. doi:10.3109/003655 18109092033

23. Caride VJ, Zubal IG (1995) Assessment of the rate of uptake-plasma volume product to calculate glomerular filtration rate. J Nucl Med 36(9):1602-1604 (corrected)

24. Mulligan JS, Blue PW, Hasbargen JA (1990) Methods for measuring GFR with technetium-99m-DTPA: an analysis of several common methods. J Nucl Med 31(7):1211-1219 